\title{
Heartbeat: Detection of arrhythmias in older adults with falls and syncope
}

\section{Catherine M Otto}

It can be challenging to identify whether an arrhythmia was the cause of a fall or syncopal event, particularly in older adults who have a high frequency of unexplained falls. Conditions such as orthostatic hypotension and vasovagal syncope are common, and many patients provide other provide plausible excuses for the fall that may or may not be correct. Diagnosis of elusive cardiac arrhythmias requires detection of intermittent infrequent events yet there has been little evidence to support widespread use of implantable loop recorders (ILR). In a detailed study using ILR in 70 patients (mean age of 70 years) with 2 or more unexplained falls, Bhangu and colleagues (see page 681) found that the fall could be attributed to a cardiac arrhythmia in $20 \%$, detected an average of 47.3 days, with a range of 1 to 190 days, after ILR implantation (figure 1). This data resulted in $14 \%$ receiving a permanent pacer for bradycardia or asystole and 6\% being treated medically for a supraventricular tachycardia. An additional 34\% of patients had modifications to their medical therapy based on an arrhythmia detected on ILR.

Although this is a small observational study, the implications of these results are profound, especially in an era when it is likely that everyone soon will be able to monitor their own heart rhythm on a daily basis. Prevention of falls is a top healthcare priority because consequences of falls include serious injuries, declining functional status, need for nursing home placement and higher use of medical resources. In an accompanying editorial, Grossman (see page 657) suggests that "using implantable loop recorders could explain the cause in over a million fallers annually in the USA alone, potentially preventing recurrent falls, improving health and functionality and substantially reducing medical cost in the geriatric population" based on the expected rate of aging of the population and the data presented by Bhangu and colleagues. No doubt the rate of diagnosis of treatable

Correspondence to Professor Catherine M Otto, Division of Cardiology, University of Washington, Seattle, WA 98195, USA; cmotto@u.washington.edu arrhythmias in older adults will increase dramatically as low-cost personal smart devices become capable of continuously monitoring heart rate, rhythm and blood pressure. Perhaps we can treat patients even before the first fall, improving quality of life and reducing health care costs.

Anticoagulation with a vitamin-K antagonist (VKA) is considered reasonable for the first 3 months after bioprosthetic mitral valve replacement or repair but there is only weak evidence supporting this recommendation, particularly after valve repair. Valeur and colleagues (see page 687) used the Danish national registry to identify 2188 patients undergoing mitral valve repair between 1997 and 2012 who had no history of stroke or previous VKA therapy, and were alive more than 1 week after hospital discharge. Compared to those not on VKA therapy, the $39 \%$ of patients discharged on VKA therapy had a lower risk of death/stroke at 3 months $(\mathrm{HR}=0.28$, CI (0.13 to 0.62$)$, $\mathrm{p}=0.002)$ with no difference in bleeding complications between the 2 groups (2\% versus 1\%). After 3 months, the incidence rates of stroke decreased significantly and were comparable in the two groups (HR $0.90(0.63$ to $1.30 ; p=0.59)$. To test the robustness of these findings, a propensityscore matched analysis of 1224 patients was examined which showed similar findings (figure 2). This study suggests that most patients should receive VKA therapy after mitral valve repair, especially in the first 3 months because atrial fibrillation is common, occurring in 25 to $40 \%$ of patients, and endothelium has not yet covered the repair site and annular ring.

The use of evidence-based guidelines for cardiac interventions and medical therapy now is firmly established. We are confident that guidelines are correct when there are statistically significant numerical data from randomized controlled clinical trials supporting each recommendation. However, these guidelines have little effect if care is not delivered effectively to

Figure 1 Flow diagram for patient screening. ILR, implantable loop recorder; SVT, supraventricular tachycardia. 

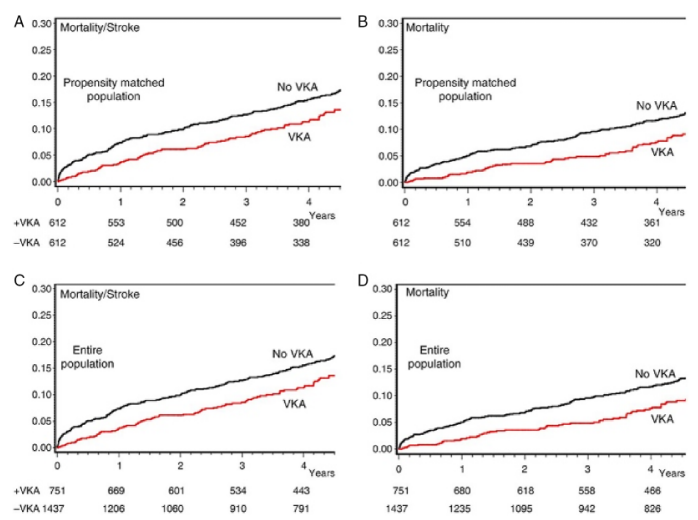

Figure 2 Kaplan-Meier curves of the combined end point mortality/stroke and of mortality according to Vitamin $\mathrm{K}$ antagonist (VKA) treatment, in the entire population and in the propensity-matched population.

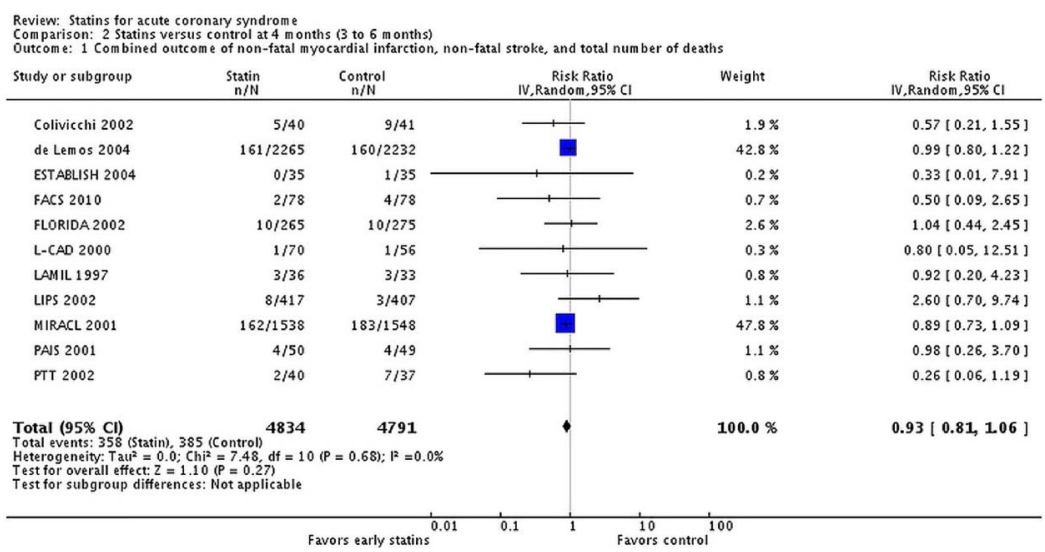

Figure 3 Forest plot of the risk of a combined outcome of non-fatal myocardial infarction, non-fatal stroke and mortality in the statin versus control groups (with permission from the Cochrane Heart Group).
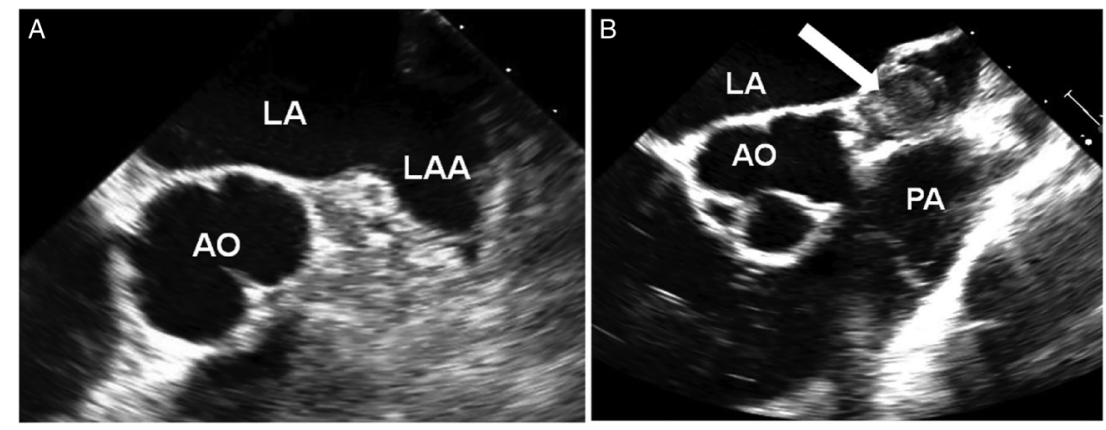

Figure 4 Two-dimensional transoesophageal echocardiography from mid-oesophageal view at $0^{\circ}(A)$ and at $60^{\circ}$ with anterior (B) flexion of the probe. AO, aorta; LA, left atrium; LAA, left atrial appendage; PA, pulmonary artery.

patients. Clark and colleagues performed a systematic review of heart failure disease management programs to identify which mechanisms of care delivery resulted in improved self-care disease management and clinical outcomes (see page 707). Based on a series of 33 studies with a total of 3344 participants, the authors conclude that effective mechanisms include professional support of self-care, interventions that increased psychosocial well-being, an increased patient understanding of the disease process, involvement of other family members in self-care, and support from health professionals in the use of technology. Technology interventions were most effective if they were portable and relatively simple to use with adequate technical support. The authors suggest these finding are hypothesis generating only; further studies examining mechanisms and outcomes of heart failure programs are needed. "This research should incorporate mixed and qualitative methods to explore systematically which mechanisms are consistently associated with positive programme outcomes and how these are moderated by other intervention and contextual factors." Given the rapid advances in low-cost portable smart devices, let's hope that young investigators perform studies using digital technology to improve health care delivery and outcomes with the same rigor we require for clinical trials of new medications or procedures.

Our Cochrane Corner feature in Heart continues in this issue with a short summary of the Cochrane Review on the clinical benefit of early statin therapy in patients with an acute coronary syndrome (see page 653). The key points for clinicians are that early statin use in ACS patients is safe, reduces risk of unstable angina at 4 months, and increases the likelihood of long-term patient adherence to statin therapy. Although there was no difference in the rate of other clinical outcome events during the randomized treatment period, it is possible that additional benefit might be seen over a longer follow-up interval (figure 3).

The Education in Heart in article in this issue focuses on management of adult with cyanotic congenital heart disease (see page 720 ). These patients face a lifetime of chronic hypoxaemia, secondary erythropoiesis, iron deficiency, hyperviscosity and coagulation issues. Even so, quality of life can maintained with cautious management in conjunction with a congenital heart disease center. Some of these patients also may benefit from pulmonary vasodilator therapy.

The Image Challenge in this issue (see page 693) provides an a elegant comparison of 2D and 3D echocardiography (Figure 4) with tomographic and 3D computed tomographic (CT) images, emphasizing the importance of spatial knowledge for interpreting cardiac images.

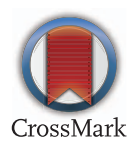

To cite Otto CM. Heart 2016;102:651-652.

Heart 2016;102:651-652.

doi:10.1136/heartjnl-2016-309648 